\title{
Paradojas Didácticas Observadas en la Gestión de los Teoremas de Euclides
}

\section{Didactical paradoxes observed in the management of Euclid's theorems}

\author{
Ismenia Guzmán Retamal ${ }^{*}$ \\ ORCID iD 0000-0002-2881-989X \\ Luis R. Pino-Fan** \\ ORCID iD 0000-0003-4060-7408 \\ Elizabeth H. Arredondo ${ }^{* * *}$ \\ ORCID iD 0000-0002-5285-1603
}

\begin{abstract}
Resumen
Los procesos de enseñanza y de aprendizaje de la geometría han permitido plantear diversos problemas que han sido estudiados intensamente por la comunidad de investigación en Didáctica de la Matemática en las últimas décadas, centrándose en aspectos tales como el uso de software, el conocimiento de los alumnos, la complejidad matemática, el conocimiento del profesor etc. No obstante, hoy en día aún sigue vigente el interés por entender las dificultades asociadas a las prácticas de los profesores, en particular, sobre la gestión de las clases que llevan a cabo, ya que esto impacta directamente en el aprendizaje de la geometría de los estudiantes. En este sentido, presentamos un estudio que consiste en la observación de la gestión de dos profesores que se han enfrentado a la enseñanza de los Teoremas de Euclides en sus cursos de segundo año de Educación Media, en Chile. Con este fin, se realizó la observación directa de las clases y, posteriormente, se entrevistó a ambos profesores. Para el análisis de la gestión pedagógica utilizamos la Teoría de Situaciones Didácticas (TSD) de Guy Brousseau, concretamente nos interesamos por las nociones de contrato didáctico, medio didáctico, procesos de devolución e institucionalización. La metodología utilizada en el estudio es de tipo cualitativa, descriptiva e interpretativa, donde se recupera extractos de episodios de una clase. El análisis revela numerosas paradojas didácticas que no favorecen el aprendizaje de los alumnos en relación con los objetivos previstos, ni a los objetos matemáticos tratados en clases. Entre las paradojas se observan fenómenos como el efecto topaze y el de resonancia, detectados en la interacción profesor/alumno. Queda en evidencia la necesidad de analizar en profundidad las prácticas profesionales de los profesores, pues en este trabajo se pone de manifiesto la ruptura entre el discurso institucional y la gestión pedagógica en práctica.
\end{abstract}

Palabras clave: Contrato didáctico. Medio didáctico. Proceso de devolución. Proceso de institucionalización. Efecto Topaze. Modelo de resonancia.

\footnotetext{
* Doctora por Universidad Louis Pasteur de Estrasburgo. Profesora Universidad de Los Lagos, Osorno, Chile. Dirección Postal: Postgrado en Educación Matemática, Calle Lord Cochrane, 1039, Osorno, Chile. E-mail: ismenia.guzman@ulagos.cl.

** Doctor por la Universidad de Granada. Profesor de la Universidad de Los Lagos, Osorno, Chile. Dirección postal: Postgrado en Educación Matemática, Departamento de Ciencias Exactas, Calle Lord Cochrane, 1039, Osorno, Chile. E-mail: luis.pino@ulagos.cl.

**** Doctora en Ciencias, especialidad Matemática Educativa por el Centro de Investigación y de Estudios Avanzados del IPN, Cinvestav-IPN México. Profesora de la Universidad de Los Lagos, Osorno, Chile. Dirección postal: Postgrado en Educación Matemática, Departamento de Ciencias Exactas, Calle Lord Cochrane, 1039, Osorno, Chile. E-mail: elizabeth.hernandez@ulagos.cl.
} 


\begin{abstract}
Geometry teaching and learning processes have allowed us to pose several problems that have been intensively studied by the Mathematics Education research community in recent decades, focusing on aspects such as software use, student knowledge, mathematical complexity, teacher knowledge etc. However, today, there is still interest in understanding the difficulties associated with the teachers' practices, in particular, regarding the management of the classes they carry out, since this directly impacts geometry learning by the students. In this sense, we present a study that consists on the observation of the management of two teachers who have faced the teaching of Euclid's Theorems in their second-year courses in Middle Education in Chile. To this end, direct observation of the classes was carried out and both teachers were subsequently interviewed. For the analysis of pedagogical management, we used Guy Brousseau's Theory of Didactical Situations (TSD), specifically focusing on the notions of didactical contract, didactical milieu, and the processes of devolution and institutionalization. The methodology used in the study is qualitative, descriptive, and interpretive, where extracts of episodes from a class are retrieved. The analysis reveals numerous didactical paradoxes that do not favor the learning of the students in relation to the foreseen objectives, nor to the mathematical objects treated in class. Among the paradoxes, phenomena such as the topaze and resonance effects are observed, detected in the teacher-student interaction. The need to analyze in depth the professional practices of teachers is evident, since this work revealed the rupture between the institutional discourse and the pedagogical management in practice.
\end{abstract}

Keywords: Didactical contract. Didactical environment. Return process. Institutionalization process. Topaze effect. Resonance phenomenon.

\title{
1 Introducción
}

En la actualidad, aunque existen diversas investigaciones orientadas a determinar el tipo de conocimientos y competencias que requiere tener un profesor de matemáticas, para gestionar adecuadamente los aprendizajes de sus estudiantes y reflexionar sobre su propia práctica tomando medidas para hacer cambios (FORTUNY; RODRÍGUEZ, 2012; LLINARES; VALLS, 2009; TCHOSHANOV, 2011; CASTRO; PINO-FAN; VELÁSQUEZ-ECHAVARRÍA, 2018; TROUCHE et al., 2019), aún hacen falta investigaciones sobre el tipo de prácticas reales que realizan los profesores en el aula y sobre los fenómenos didácticos que surgen de tales prácticas.

Los resultados de estas investigaciones podrían generar pistas sobre el tipo de procesos formativos requeridos para ayudar al profesorado a superar las falencias y optimizar sus prácticas didáctico-matemáticas en beneficio de los aprendizajes de los estudiantes. En este sentido, el objetivo de nuestro artículo es presentar evidencia empírica de la emergencia de paradojas didácticas en los procesos de enseñanza y de aprendizaje de las matemáticas, concretamente en un proceso de enseñanza y de aprendizaje sobre los Teoremas de Euclides. Con ello, buscamos que el profesorado reconozca y tome conciencia de la existencia de estas paradojas, las cuales dificultan un adecuado aprendizaje por parte de los estudiantes.

Este estudio se inscribe en la línea de investigación de formación de profesores relacionada con las prácticas docentes, y consiste en un análisis de la gestión de clase sobre los Teoremas de Euclides. A partir de la observación directa de clases reales (sin intervención 
de los investigadores), hemos realizado el protocolo de cada una de ellas; seleccionamos la primera parte de la clase referida a la introducción del tema y algunos ejemplos o ejercicios con los que se desarrolla el tema previsto. Las informaciones recogidas se han complementado con entrevistas individuales a los profesores, con el fin de conocer sus posturas en relación con el saber escolar, los objetivos en juego, sus métodos pedagógicos y la gestión de los tiempos para el proceso de enseñanza y aprendizaje del objeto matemático tenido en cuenta. El estudio se inscribe en la metodología de investigación cualitativa.

La Didáctica de las Matemáticas (DM) puede ser entendida como ciencia de tipo explicativo, o bien de tipo comprensivo, dando respuesta a dos demandas diferentes (BREDA; FONT; PINO-FAN, 2018). La primera demanda pretende que los constructos teóricos de la DM sirvan para comprender los procesos de enseñanza-aprendizaje de las matemáticas y, la segunda, que estos sirvan para guiar la mejora de dichos procesos. En este sentido, en este artículo usamos el término paradojas didácticas para referirnos a todas aquellas ideas, acciones o expresiones del profesor que van en contra de su misión de favorecer los aprendizajes de los estudiantes.

Para esto, adoptamos el marco de la Teoría de Situaciones Didácticas (TSD) de Guy Brousseau (1986, 1988a, 1988b, 1990, 1995, 1998, 2000, 2002, 2008), en donde este tipo de fenómenos son atendidos, empleando las nociones de medio y contrato didáctico, esenciales para abordar la gestión real del docente en el aula; así mismo, se consideran los procesos de devolución e institucionalización como responsabilidades fundamentales del docente durante su gestión, pues a partir de las diferentes herramientas de esta teoría podemos dar cuenta de dichas contradicciones, entre otros fenómenos.

Con ayuda de estas nociones, se analizan los protocolos de las clases para identificar algunos fenómenos didácticos que se desprenden de la interacción múltiple que ocurre en el sistema didáctico, en particular aquella del docente y los alumnos frente al saber matemático en juego. Para el análisis de la interacción profesor-alumno en clase, nos apoyamos en el modelo de resonancia, el cual se refiere a la intervención de los alumnos en el proyecto del profesor (COMITI; GRÉNIER; MARGOLINAS, 1995).

En el desarrollo de este estudio, primero presentamos la selección de cada protocolo y el análisis respectivo. En segundo lugar, mostramos un extracto de las entrevistas, seguido de observaciones, con el fin de cruzar las informaciones obtenidas tanto de la observación de clase como de las entrevistas. Siguen, a continuación, algunos comentarios finales y eventuales prolongaciones de la investigación. 


\section{Teoría de Situaciones Didácticas}

En la teoría de Situaciones Didácticas de Guy Brousseau, son esenciales las nociones de contrato didáctico y medio didáctico. El primero describe el conjunto de comportamientos (específicos) del profesor que son esperados por el alumno y el conjunto de comportamientos del alumno que son esperados por el profesor. Esta noción permite distinguir dos tipos de situaciones estrechamente vinculadas, las ligadas al aprendizaje (denominadas adidácticas) y las ligadas a la enseñanza (denominadas didácticas). En particular, para las situaciones adidácticas se identifica una actividad (problema, tarea o preguntas matemáticas), un medio didáctico que permite poner en escena la actividad y un contrato didáctico que regula la gestión de la actividad del profesor en la clase. A medida que avanza la clase, el medio y el contrato entran en relación. El medio puede hacer cambiar el contrato, y viceversa (BROUSSEAU, 1986, 1990, 1998; MARGOLINAS, 1992, 1993， 1995; PERRINGLORIAN, 1994; HERSANT, 2014).

En síntesis, se entiende por medio didáctico todo aquello que actúa sobre el alumno y aquello sobre lo cual el alumno actúa. Es un medio antagonista, dice Brousseau, ya que interpela al alumno y este reacciona. El medio puede ser material (papeles, tijeras, reglas, compás, computador etc.) o intelectual (definiciones, propiedades, teoremas, convenciones sobre notaciones, símbolos matemáticos).

El contrato didáctico surge como una necesidad teórica con el fin de comprender el mal funcionamiento o irregularidades de los procesos de aprendizaje. Él hace la conexión entre las situaciones adidácticas y didácticas, y pone en evidencia el proceso de devolución (BROUSSEAU, 1998). En general, el contrato didáctico es implícito para el profesor, pero el investigador, con frecuencia, puede identificarlo en la gestión de un profesor en el aula. Esencialmente, tiene que ver con el proyecto de enseñanza del profesor, en particular con sus metas y la metodología de la clase que realizará, la responsabilidad que dejará a los alumnos para responder en forma autónoma a sus preguntas o propuestas de tareas (LAPARRA; MARGOLINAS, 2011).

Por otra parte, el contrato didáctico permite observar el disfuncionamiento del proceso de enseñanza y de aprendizaje. Cuando alguno o algunos de los elementos del proceso de enseñanza fracasa en la gestión del profesor, este, para no reconocer su fracaso, intenta salvar la apariencia de un aprendizaje de diferentes maneras; es aquí cuando se manifiestan las paradojas (BROUSSEAU, 2002). Es necesario señalar que el contrato didáctico es incierto, en tanto que el profesor no puede asegurar el éxito del aprendizaje de todos sus alumnos. Las 
paradojas son consecuencia de la incertidumbre del contrato didáctico.

Para Brousseau (2002), una paradoja no es por completo una contradicción, sino un obstáculo en la consecución de aprendizajes. De hecho, Brousseau señala que estas constituyen las piedras de tope de toda teorización de los fenómenos didácticos. De este modo, los efectos (Topaze, Jourdain, Deslizamiento meta-cognitivo y otros) señalados por Brousseau, son paradojas didácticas, cuyas características y usos deberían ser conocidos por el profesorado de matemáticas, con la finalidad de evitarlas y mejorar, así, la gestión de los procesos de enseñanza y de aprendizaje de esta disciplina.

Los efectos que se presentan durante la interacción en una situación didáctica pueden interrumpir o inhibir la construcción del conocimiento que lleva el estudiante, siendo estos efectos negativos dentro del proceso de enseñanza-aprendizaje (BROUSSEAU, 2002). En seguida presentamos una descripción breve de estos efectos:

a) Efecto Topaze o control de la incertidumbre, se caracteriza por que el estudiante llega a la solución de una situación dada apoyado por medios externos; esto significa que, ante una situación dada, el profesor evita la elaboración de una respuesta por parte del alumno, al darle señales para que el alumno dé la respuesta que él espera.

b) Efecto Jourdain, considerado también como el malentendido fundamental, consiste en que el docente reconoce un índice de conocimiento en la respuesta del alumno, la cual está asociada a un conocimiento banal, que el docente valora como un conocimiento válido, aunque la respuesta del alumno sea incorrecta o corresponda a otro objeto.

c) Deslizamiento Metacognitivo, generalmente se presenta cuando un profesor tiene dificultades para lograr la comprensión y el logro de los alumnos de un objeto matemático que ha previsto; entonces, cambia el objetivo de su enseñanza y lo reduce a la enseñanza de un objeto de menor nivel cognitivo o al manejo de una técnica.

d) El abuso de la analogía, se refiere a que el profesor suplanta el estudio de una noción matemática, recurriendo a un caso particular con alguna similitud del objeto, pero que dista de la concepción misma del objeto de estudio. Es necesario que se tenga cuidado con el uso de las analogías, ya que es un medio que favorece la producción de efectos Topaze.

e) El envejecimiento de las situaciones de enseñanza, sucede cuando el profesor recurre a la reproducción de una clase que ya ha preparado, en forma natural 
intentará hacer cambios, los cuales pueden perder la riqueza inicial del objeto de estudio, pues el profesor no considerará u olvidará algunos de los aspectos esenciales del objeto, en el fondo estos cambios pueden empobrecer su esquema original. Mientras más veces intente el profesor reproducir una clase, esta será cada vez más pobre.

En la TSD, el proceso de devolución cobra una gran importancia puesto que puede evitar el efecto Topaze. Brousseau (1998, p. 92), señala que este efecto consiste en que el "profesor da señales al alumno para que responda aquello que él espera, pero que el alumno no ha producido por sí mismo; no obstante, el profesor la acepta como resultado de un aprendizaje o la comprensión de un hecho". En el proceso de devolución "el alumno debe estar situado en posición didáctica o seudo didáctica".

Las situaciones de institucionalización, Brousseau las describió en la primera escuela de verano en Francia (École d'été 1980) como

[...] aquellas situaciones en las cuales se fija convencionalmente y explícitamente el estatus cognitivo de un conocimiento y un saber. La institucionalización se dice interna si un grupo fija libremente sus convenciones según un proceso cualquiera de acuerdo a un sistema cuasi aislado. Por otro lado, se dice externa si las convenciones son acordes a una cultura o a una institución, es la situación didáctica más frecuente (BROUSSEAU, 1988, p. 282).

Brousseau, en esa época, distinguía entre las nociones de saber y conocimiento, las que ha precisado en el proceso de institucionalización; él señalaba que en el estudio de los medios de comunicación, un saber comienza por la identificación de las manifestaciones de conocimientos relativos a ese saber en una comunidad, y prosigue con definiciones $u$ observaciones de comportamientos que manifiestan la adquisición de ese saber en sujetos pertenecientes a esa comunidad.

De este modo, el saber funciona como medio cultural que permite clasificar, describir y comunicar los conocimientos y las prácticas adquiridas. Fundamentalmente, la institucionalización va a permitir reconocer lo que debe saberse, va a clarificar el saber al cual, posteriormente, se puede hacer referencia, es decir, el saber oficial o saber institucional.

En relación con la interacción en situación de clases, Comiti, Grénier y Margolinas (1995) describen el fenómeno de resonancia de la intervención o no de los alumnos en el proyecto del profesor. Las autoras distinguen niveles de resonancia: Resonancia Fuerte (ante la intervención errónea de algún alumno, el profesor se hace cargo de esta, planteando un escenario de discusión, hasta que el error queda dilucidado); Débil (cuando la participación e interacción del profesor es solo con algunos estudiantes); Insuficiente o Nula (cuando hay intervención, correcta o incorrecta, de uno o algunos alumnos que sacan al profesor de su 
discurso, este no la toma en cuenta). A continuación, en el siguiente apartado, se presenta la metodología implementada en este estudio; posteriormente, el análisis de los protocolos de las sesiones seleccionadas y de las entrevistas de acuerdo al marco teórico ya señalado.

\section{Metodología}

En esta investigación se utiliza una metodología de tipo cualitativa, descriptiva e interpretativa, para desarrollar un análisis exhaustivo del desarrollo de las clases y del cruce de información con las entrevistas.

Los sujetos de estudio son dos profesores de segundo medio, bien calificados por parte del Ministerio de Educación en Chile, uno de ellos posee el grado de Magíster. Ambos trabajan en liceos municipales que reciben subsidio del Ministerio de Educación, de manera especifica, los libros de texto utilizados por ellos son proporcionados por la entidad. La población a la que atienden se encuentra en desventaja académica por el medio socio-cultural al que pertenecen los alumnos, es decir, este tipo de colegios públicos en Chile se consideran vulnerables.

El diseño metodológico consta de dos instrumentos: el protocolo de clases y la entrevista. El primero consiste en la crónica del discurso en la clase recogida, en una observación directa no participante de esta. Se diseñan tablas de dos columnas, una para describir paso a paso las acciones del profesor y su interacción con el grupo de estudiantes, la segunda contiene el análisis y detección de los fenómenos observados durante la clase. Este protocolo se divide en episodios, los cuales se determinan por las acciones claves desarrolladas en la clase por el profesor o por algún alumno. Cabe destacar que cada episodio responde al objetivo, explícito o no, del profesor o a una intervención de un alumno que necesite atención.

La clase observada corresponde al tema de Teoremas de Euclides, presente en las matemáticas de segundo grado de Educación Media, en Chile. En el programa de estudio de Matemáticas de Segundo Medio, en la Unidad 2 de Geometría, el Ministerio de Educación (MINEDUC, 2011, p. 37) plantea como un aprendizaje esperado: "Demostrar los teoremas de Euclides relativos a proporcionalidad de trazos", ligado con los siguientes objetivos: 1) conocer los Teoremas de Euclides, y 2) aplicar los Teoremas de Euclides.

La entrevista, que se lleva a cabo con los profesores, consta de doce preguntas abiertas que tienen por objeto recopilar información sobre la labor pedagógica, y considera los siguientes aspectos: los elementos considerados en la gestión de clase, los materiales de apoyo 
(libros o materiales), el valor dado al contenido matemático a desarrollar, las actividades o tareas que se propongan, entre otros. El objetivo fundamental de esta entrevista es complementar las informaciones obtenidas en el protocolo y facilitar el cruce de información entre la práctica docente y con su discurso institucional.

\section{Presentación de protocolos y análisis respectivos según la TSD}

A continuación, mostramos algunos episodios extraídos de las clases realizadas por dos profesores de Educación Media que designamos por A y B. Estos serán comentados sobre la gestión del profesor y describen acciones de alguno de los actores o interacciones entre el profesor y los alumnos. Señalamos por P las intervenciones del profesor, por Ai las intervenciones individuales de los alumnos, y por Als las intervenciones grupales de los alumnos. En cada episodio enumeramos las intervenciones importantes de la clase.

\subsection{Comentarios sobre la gestión del profesor $\mathrm{A}$}

La gestión del profesor A deja en evidencia que su metodología de clase es expositiva, ya que al interactuar con los alumnos formula preguntas superficiales y de respuestas cerradas. El ejemplo que hemos analizado es un problema rutinario de aplicación de una fórmula que corresponde a la representación numérica del Teorema de Euclides relativo al cateto, como se observa enseguida en la Figura 1. 


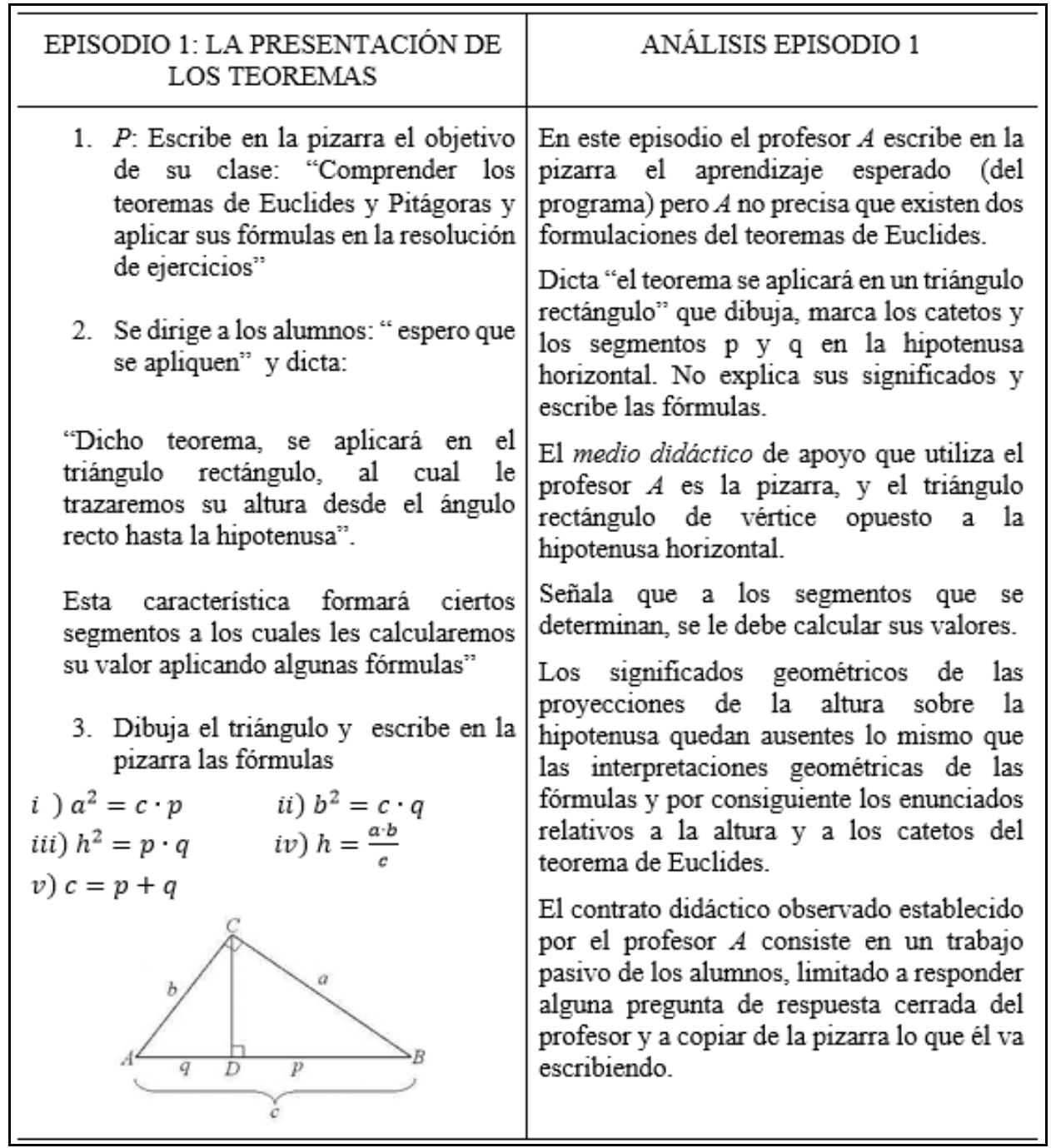

Figura 1 - Extracto del episodio 1 de la Clase de profesor A y su análisis según la TSD Fuente: elaborada por los autores

Desde el punto de vista del saber matemático, la clase resulta incompleta. En primer lugar, el profesor señala que trabajará con un triángulo de posición típica, un triángulo rectángulo con el vértice del ángulo recto opuesto a la hipotenusa horizontal; esta posición del triángulo expresa, será siempre la misma y lo único que cambiará serán los valores y el tipo de letra, instante (2) del Episodio 2, como se observa enseguida en la Figura 2.

\begin{tabular}{|c|c|}
\hline EPISODIO 2: UN EJEMPLO & ANÁLISIS EPISODIO 2 \\
\hline $\begin{array}{l}\text { 1. P: Oralmente se dirige a los alumnos: } \\
\text { "El ejemplo dice lo siguiente, después lo } \\
\text { Anotan. Tenemos el triángulo, donde } \\
q=6, c=8 \text { Cuánto mide } a \text { ?" } \\
\text { 2. P: "Siempre vamos a trabajar en este triángulo } \\
\text { y vamos a ir variando los valores y el tipo de } \\
\text { letra que me estén pidiendo". }\end{array}$ & $\begin{array}{l}\text { El Profesor propone un ejercicio que contiene un } \\
\text { cateto y las proyecciones sobre la hipotenusa pero } \\
\text { no enfatiza en las interpretaciones geométricas de } \\
\text { esos segmentos ni en los enunciados del teorema. } \\
\text { El Profesor ha dicho que van a trabajar siempre en } \\
\text { el mismo triángulo, es decir el que ha dibujado en la } \\
\text { pizarra, el triángulo rectángulo de base horizontal y } \\
\text { considerándolo propone el ejercicio y lo va } \\
\text { solucionando en una suerte de diálogo con algunos } \\
\text { alumnos, aquellos que siguen su procedimiento. A } \\
\text { partir de aquí la clase consiste en hallar el valor que } \\
\text { falta en una expresión. }\end{array}$ \\
\hline
\end{tabular}

Figura 2 - Extracto del episodio 2 de la Clase de profesor A y su análisis según la TSD Fuente: elaborada por los autores 
El profesor A tampoco enuncia el teorema, limitándose a escribir expresiones simbólicas en la pizarra. De esta manera, el medio didáctico que ha puesto en juego es pobre, pues no presenta ningún desafío, no interpela a los alumnos, no es un medio antagonista. Las preguntas que plantea el profesor A son superficiales y, aun así, cuando hubo alumnos que respondieron o preguntaron, el profesor no los tomó en cuenta. Por otra parte, las ecuaciones presentadas no las ha interpretado geométricamente, dejándolas en un ámbito numérico sin relación con los objetos geométricos correspondientes. En consecuencia, el profesor A no ha favorecido una oportunidad de aprendizaje.

El estilo de clase no ha dado oportunidad para que los alumnos realizaran algún trabajo autónomo o les permitiera realizar cuestionamientos. Por otra parte, ha quedado en evidencia el fenómeno de resonancia nula, el cual se relaciona con la interacción entre los alumnos y el profesor; en este caso, ha habido ausencia de resonancia, un ejemplo de este fenómeno se ha observado en las intervenciones (9) y (11) del episodio 3, cuando la intervención del alumno A5 no fue tomada en cuenta. La ausencia de resonancia deja en evidencia que el profesor A no ha favorecido la interacción con él, y tampoco se ha hecho cargo de atender la pregunta del alumno A3 en el instante 6 del episodio 1, lo que significa también que él no dio lugar al proceso de devolución (Figura 3). 


\begin{tabular}{|c|c|}
\hline EPISODIO 3 :RESOLUCIÓN DEL EJERCICIO & ANÁLISIS DEL EPISODIO 3 \\
\hline 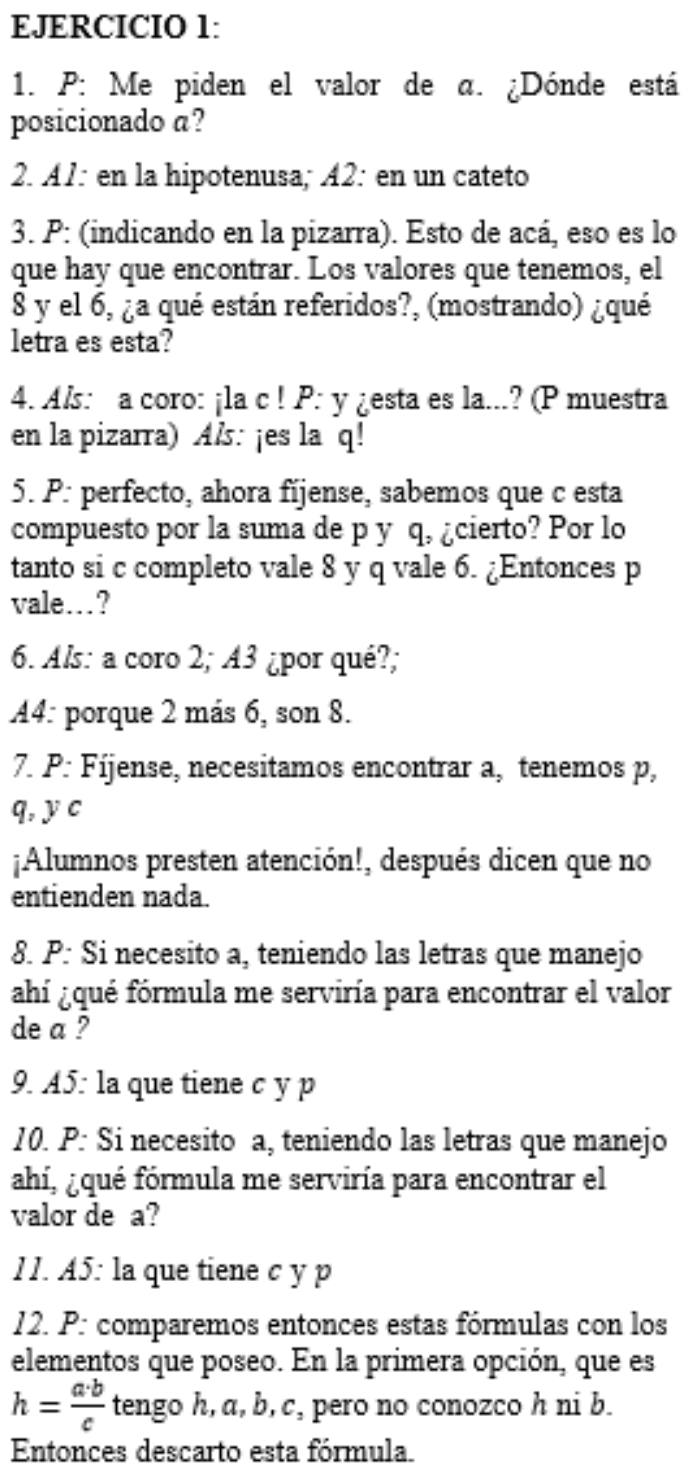 & $\begin{array}{l}P \text { va indicando paso a paso el procedimiento de } \\
\text { resolución. Ante la pregunta sobre la posición de } a \\
\text { en el triángulo, dos alumnos } A 1 \text { y } A 2 \text { responden. } \\
\text { Pero el profesor no toma en cuenta sus respuestas. } \\
\text { Instante } 1 \text {. Se produce entonces el fenómeno } \\
\text { didáctico llamado, fenómeno de Resonancia nula. } \\
\text { Entre el instante } 3 \text { y el } 6 \text {, las preguntas de } P \text { son poco } \\
\text { significativas y las respuestas corresponden al } \\
\text { fenómeno del Efecto Topaze. } \\
\text { La pregunta de A3, P no la toma en cuenta. } \\
\text { Y necesitaba atención, puesto que la respuesta que da } \\
\text { A4, tal vez no aclaraba la duda de A3. } \\
\text { Como solo algunos alumnos siguen el procedimiento } \\
\text { de } P \text {, él se ve obligado a llamarles la atención al resto } \\
\text { del curso y sigue con su línea de desarrollo. }\end{array}$ \\
\hline
\end{tabular}

Figura 3 - Extracto del episodio 3 de la clase de profesor A y su análisis según la TSD Fuente: elaborada por los autores

Respecto del contrato didáctico, el cual es implícito y se planifica durante la preparación de la clase, no se ve presente en la gestión de esta y solo se logran apreciar las tareas previstas para los alumnos por parte del profesor, el cual no ha considerado posibles dudas emergentes durante la sesión de clase. En este caso, el profesor A no ha previsto tareas distintas a la de responder sus preguntas (superficiales y de respuesta cerradas), así que la responsabilidad que el docente asigna a los estudiantes consiste en poner atención a la resolución del ejercicio que él realiza en la pizarra. La clase continúa con otros ejercicios y con la misma modalidad. 


\subsection{Análisis de la gestión de la profesora B según la TSD}

La profesora B elige un medio didáctico que consiste en: diapositivas Power Point en donde presenta ejercicios resueltos, la pizarra y los cuadernos de los alumnos. El contrato didáctico que B establece para su clase es de gestión expositiva, las acciones del profesor son de lectura y de reescritura en la pizarra, reproduciendo las diapositivas. Las explicaciones están en un lenguaje confuso, la profesora B hace afirmaciones que no explica, por ejemplo, en el episodio 1 (Figura 4), habla de valores proporcionales sin precisar a qué se refiere.

\begin{tabular}{|c|c|}
\hline EPISODIO 1 : LA PRESENTACIÓN DE LOS TEOREMAS & ANÁLISIS EPISODIO 1 \\
\hline $\begin{array}{l}\text { P: Lo importante aquí chicos es que distingan los catetos, } \\
\text { hipotenusa y la altura, ¿Por qué? Porque con estas } \\
\text { fórmulas básicas más el teorema de Pitágoras vamos a } \\
\text { sacar los valores de los distintos segmentos en los } \\
\text { triángulos rectángulos que ahí se forman. } \\
P \text { : Tienen ahí dos triángulos, el ACB es exterior, acá se } \\
\text { indica que a es el cateto } \mathrm{BC} \text {, que b es el cateto AC y la } \\
\text { hipotenusa es } \mathrm{AB} \text {. Aqui } \mathrm{h}_{\mathrm{c}} \mathrm{el} \text { segmento DC, es la altura. } \\
\text { AD es q y DB es p, son las proyecciones de la altura } \\
\text { sobre la hipotenusa. } \\
\text { Tenemos el segmento } \mathrm{q} \text {, es decir, de A hasta D es la } \\
\text { proyección del cateto B sobre la hipotenusa, es decir, } \\
\text { esos valores son proporcionales y el valor de p, que es el } \\
\text { otro pedacito de acá, también es la proyección del otro } \\
\text { cateto sobre la hipotenusa. }\end{array}$ & $\begin{array}{l}\text { En este episodio, la profesora } B \text { presenta la diapositiva } \\
\text { adjunta, la describe: } \\
\text { La profesora B no señala explícitamente el objetivo. No } \\
\text { obstante éste sería obtener los valores de los segmentos que } \\
\text { se forman la hipotenusa y los lados. Esto se constata a } \\
\text { través de los ejercicios que propone. } \\
\text { E1 medio didáctico de apoyo que ha elegido se compone de } \\
\text { las diapositivas que ha preparado y que exhibe, en ellas: el } \\
\text { triángulo típico con el ángulo recto opuesto a la hipotenusa } \\
\text { horizontal. } \\
\text { E1 Contrato Didáctico como ya mencionamos, es implicito } \\
\text { pero es posible observarlo a través de las acciones del } \\
\text { profesor y de las tareas que propone a los alumnos durante } \\
\text { la clase. } \\
\text { La profesora B realiza en voz alta lecturas de los contenidos } \\
\text { de las diapositivas. La tarea para los alumnos es poner } \\
\text { atención, copiar de las diapositivas lo escrito y responder a } \\
\text { sus preguntas, que resultan superficiales y de respuesta } \\
\text { cerrada. De manera que el Medio didáctico resulta limitado } \\
\text { y sin posibilidades para los alumnos de elaborar por si } \\
\text { mismos alguna respuesta. }\end{array}$ \\
\hline
\end{tabular}

Figura 4 - Extracto del episodio 1 de la clase de la profesora B y su análisis según la TSD Fuente: elaborado por los autores

La misma dinámica se identifica en el episodio 2 (Figura 5), donde la profesora B trata de recuperar algunos elementos que le servirán de referentes para establecer elementos que apoyen la solución de algunos problemas.

En el episodio 2 hay dos diapositivas, la primera tiene como título Teoremas de 
Euclides; mientras que la segunda no lo tiene. En la primera presenta dos enunciados del teorema, el relativo a la altura y el correspondiente a los catetos. Desde el punto de vista de la TSD, las diapositivas 1 y 2 corresponden a la institucionalización del saber, el cual matemáticamente está presentado en forma imprecisa, la interpretación geométrica de las fórmulas está ausente, así como la explicación de dónde surgen ellas.

Entonces, se puede preguntar: ¿qué significado tienen esas fórmulas para los alumnos?, ¿qué aprendizaje podrían lograr ellos?, ¿cuál es el aprendizaje que ha previsto la profesora $\mathrm{B}$ ?

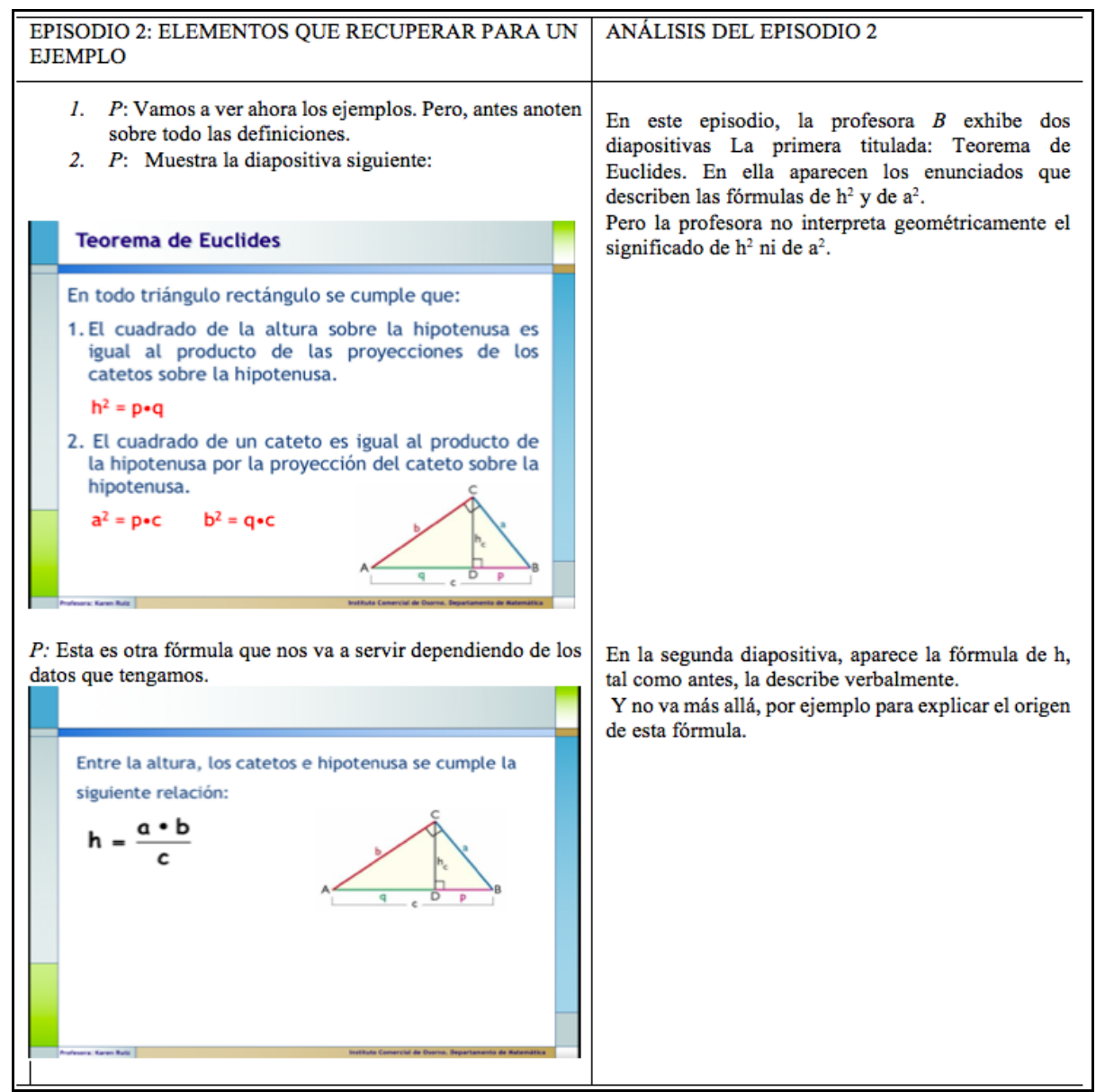

Figura 5 - Extracto del episodio 2 de la clase de la profesora B y su análisis según la TSD Fuente: elaborada por los autores

La profesora B no ha previsto tareas para sus alumnos, limitándose a lo escrito en las diapositivas. Algunos alumnos no entienden la dinámica, tal como se observa en la Figura 6. 


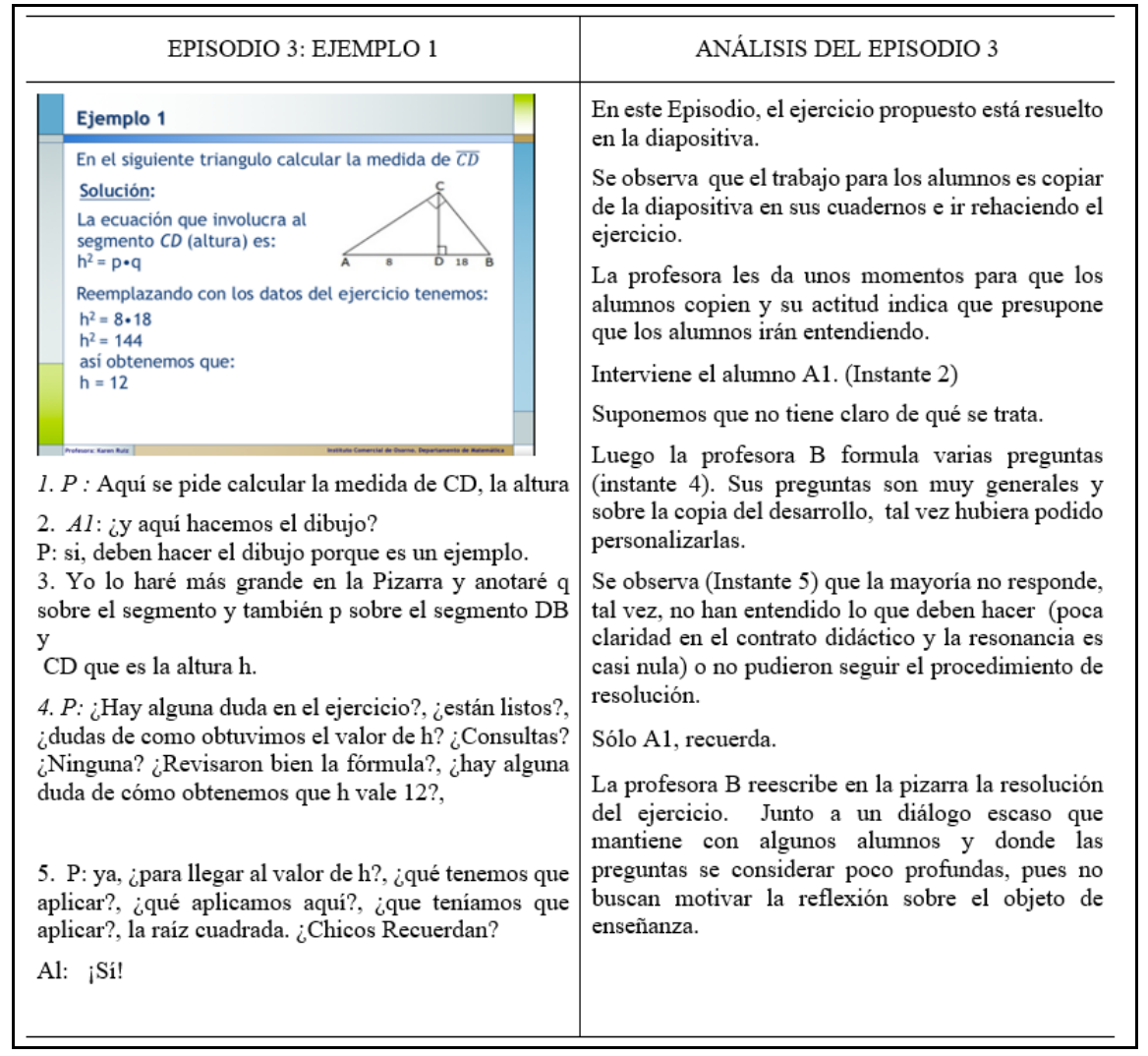

Figura 6 - Extracto del episodio 3 de la clase de la profesora B y su análisis según la TSD Fuente: elaborada por los autores

Tal vez, la profesora B contaba con que los alumnos seguirían su resolución de la diapositiva proyectada en la pizarra, pero solo algunos alumnos la seguían, y cuando surgía alguna respuesta equivocada, no la tomaba en cuenta. Este esquema de clases ha dejado en evidencia los fenómenos didácticos del efecto topaze y el de ausencia de resonancia, al no haber una verdadera interacción con los alumnos.

En el ejemplo 2 del episodio 4 (Figura 7), a partir de la figura inicial y las proyecciones $p$ y $q$, se pide encontrar la altura y los lados del triángulo $\mathrm{ABC}$. La profesora $\mathrm{B}$ explica:

Aquí independientemente de cómo se vea la figura no tenemos que guiarnos por el aspecto de la figura. Tenemos que guiarnos por los datos que nos dan, el dibujo es solo referencia (Profesora B, 2017).

Esta afirmación muestra que, para la profesora B, los Teoremas de Euclides responden a cálculos algebraicos y no aprovecha las representaciones algebraicas para conectarlas con las figuras geométricas de cuadrados, rectángulos y sus superficies. 


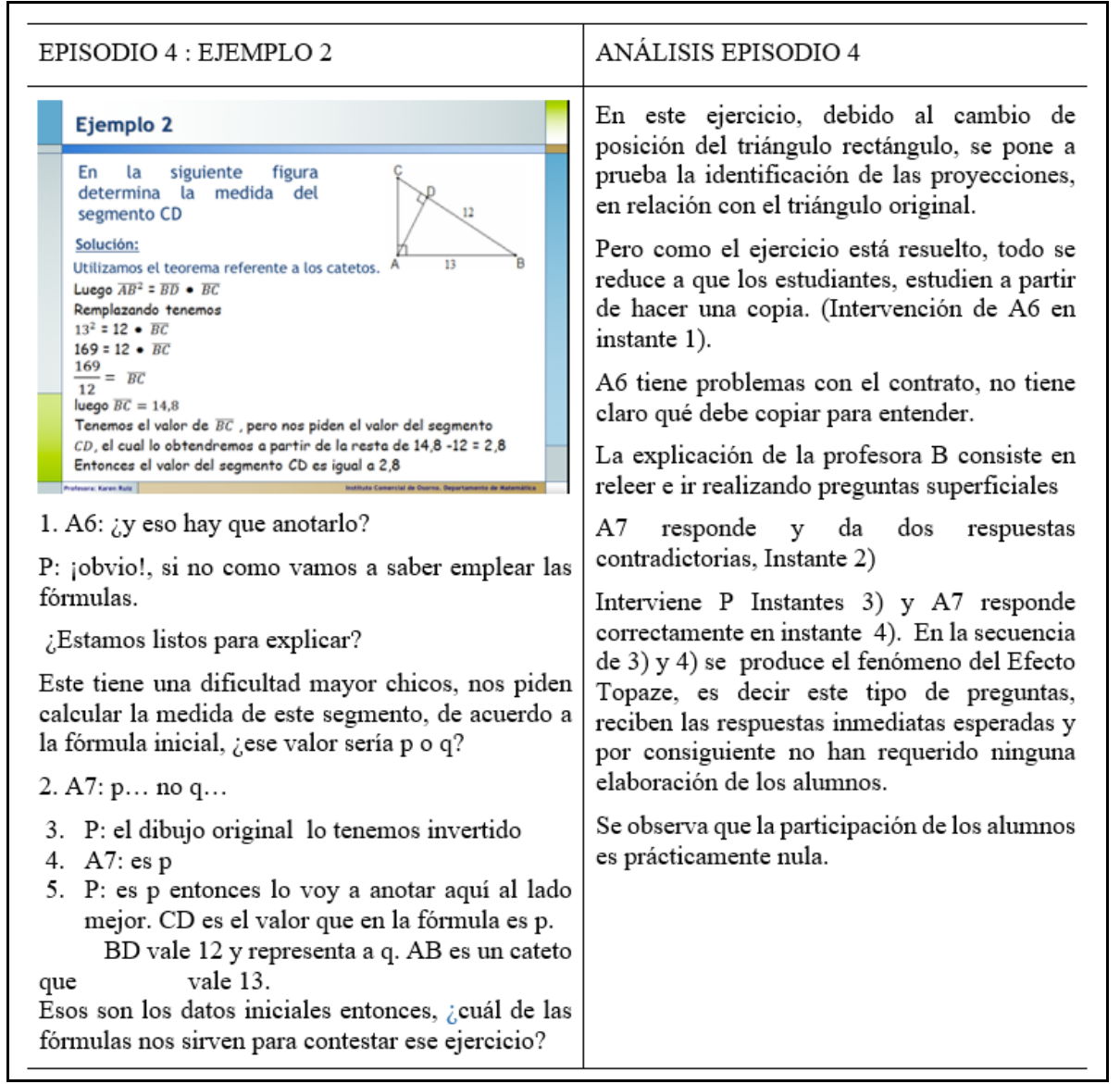

Figura 7 - Extracto del episodio 4 de la clase de la profesora B y su análisis según la TSD Fuente: elaborada por los autores

De esta manera el saber matemático-geométrico queda desperfilado, ya que la profesora B ha puesto el énfasis en la aplicación de las fórmulas.

\section{Extractos de las entrevistas y su análisis}

Para el análisis de las entrevistas, hemos tomado las ideas principales expuestas por los profesores. Designamos por $\mathrm{E}$ al entrevistador y por $\mathrm{P}$ al profesor entrevistado.

\subsection{Entrevista al Profesor A}

1. E: ¿Cuáles son los objetivos que usted se ha planteado para esta clase sobre los Teoremas de Euclides?

P: En primera instancia, creo que es importante como objetivo, el recordar los conceptos básicos y previos que están asociados al Teorema de Euclides. El recuerdo se hace a través de clase expositiva, haciendo alusión a algún ejemplo, algún problema o algún ejercicio puntual. Posteriormente, sería el definir los conceptos y después 
ejemplificar y practicar.

2. E: En el programa de segundo medio, actualizado el año 2011, aparece como objetivo Demostrar el Teorema de Pitágoras y los de Euclides, pero usted no aborda la demostración. ¿Por qué?

P: En Chile, como todos sabemos, la educación no está en un mismo nivel para todos. En el curso en que estoy trabajando, no hay espacio para la demostración de los alumnos ya les cuesta el contenido.

3. E: ¿Considera que los Teoremas de Euclides son un contenido primordial y obligatorio dentro del programa de segundo medio?

P: Es necesario, de hecho, el Teorema de Euclides junto con el de Pitágoras es una de las cosas más tangibles dentro de la matemática, ya que, si uno dice Teorema de Euclides, Teorema de Pitágoras, es algo conocido para los alumnos, no así si digo parábola, ellos son más comunes.

4. E: ¿Cuántas horas en promedio cree que se le debe dedicar a este tema?

P: Este también es un tema bastante relativo en mi modo de clase, creo que entre 7 a 10 horas repartidas entre el Teorema de Euclides y Pitágoras, el recordatorio seguido de una buena práctica con guía de ejercicios.

5. E: En cuanto a libros ¿usa alguno en específico? ¿Tiene algún libro preferido?

P: He visto libros de Santillana que son bastante buenos, pero lamentablemente el alumno debe comprarlos, los colegios particulares los usan, pero como no estoy en un colegio particular, los alumnos no tienen los medios para comprar estos libros. Uno tiene que enfocarse a los libros que entrega el ministerio, pero no los utiliza, porque el material que contienen no es adecuado para abordar lo que uno quiere. Creo que la principal falencia de los libros del ministerio, así como también de algunas pruebas estandarizadas, es la ambigüedad en el planteamiento de los ejercicios o las situaciones problemáticas, creo que la ambigüedad confunde más al alumno y obviamente también al profesor. En general, para preparar mis clases, guías o lo que sea, yo mismo construyo los ejercicios o busco ejercicios en internet, los cuales reviso para verificar si están bien estructurados y son acordes a las necesidades de los estudiantes.

6. E: ¿Qué método de enseñanza emplea para tratar los Teoremas de Euclides? ¿Es el mismo que utiliza para los otros contenidos?

P: Yo creo que la manera que a mí me ha dado resultado, es una clase expositiva, estructurando bien todo para que los contenidos no queden en el aire. Posteriormente 
la resolución de ejercicios con distintas variaciones en los elementos.

7. E: En cuanto a la realización de sus clases, ¿considera que está en un ambiente propicio como a usted le gustaría o piensa que le falta material, le falta tiempo, instrumentos didácticos?

P: Desde el punto de vista didáctico, de la infraestructura, de los materiales, yo creo que no hay un mayor problema, creo que las guías que uno necesita las tiene cuando las requiere, creo que el material que uno quiere en general está. El problema en relación con el ambiente propicio puede radicar algunas veces en el comportamiento de los alumnos. Como explicaba anteriormente, la mayoría de ellos [los alumnos] no están muy interesados por estudiar y esto hace que se genere desorden en clases. Lo que no favorece un ambiente propicio para enseñar, entonces algunas veces las clases no salen como uno quisiera.

8. E: ¿Cómo entiende el proceso de institucionalización?

P: Es algo que uno realiza por inercia, es lo que hace en la asignatura, uno parte de la definición formal, estructura los ejercicios y todo. Hay veces en que los alumnos trabajan con entusiasmo. Al momento del resumen en la pizarra, les digo: miren de dónde partimos, miren por dónde pasamos y miren dónde terminamos. Quedan [los alumnos] oh de veras, y no era tan difícil.

9. E: ¿Qué importancia le otorga a la resolución de problemas en el aula?

P: Creo que es fundamental, de hecho, la resolución de problemas es lo que a los matemáticos nos lleva a crear el pensamiento abstracto. La mecanización la puede hacer cualquiera, pero la resolución de problemas es lo que realmente nos crea ese pensamiento lógico matemático. Ahora, hay que tener en cuenta el nivel del problema, el curso al que se está aplicando, el tipo de los alumnos.

10. E: ¿Qué tipo de problemas prefiere abordar en clases? ¿Rutinarios o abiertos?

P: Yo prefiero trabajar con los más rutinarios, pero que el problema sea atractivo para los alumnos. De hecho, por ejemplo, recuerdo haber hecho problemas del tipo: Pablito tenía tantas manzanas, donde Pablito es un compañero de curso, entonces los chicos se sienten identificados con el problema. Ahora, si siento que el contenido da para un poquito más, o hay algún alumno motivado o interesado, uno puede entregar algo un poco más difícil.

11. E: ¿Cómo controla el aprendizaje de los alumnos durante la clase?

P: Lo que me dio resultado este último tiempo fue el trabajo en pequeños grupos, donde por ejemplo después de una ejercitación previa decía, ¡ya chicos!, vamos a 
trabajar este ejercicio, el grupo que lo resuelva primero, o el que lo explique va a tener dos décimas que se van a ir acumulando para el grupo y para la prueba mensual. Entonces, se trata de dejar ejercitar un momento y después empiezo a preguntar y trato de localizar dónde están los focos de conversación. Se genera el debate con base en lo que ellos opinen y argumentan, veo quién tiene la razón de reconducir cuando están errando. Yo creo que ese es el método.

12. E: ¿Qué lenguaje usa con los estudiantes? ¿Lenguaje matemático, natural o mixto?

P: En realidad soy bastante mixto, siento que los chicos no van a aprender si yo hago todo formal y ocupo pura simbología. Siento que hay que dividir el lenguaje para que los chicos no se sientan del todo ajenos a ellos, por ello trato de ser coloquial. Pero posteriormente hay que formalizar, porque la matemática tiene una estructura formal, entonces tiene que haber un matiz entre uno y otro.

\subsection{Entrevista a la Profesora B}

1. E: ¿Cuáles son los objetivos que usted se ha planteado para esta clase sobre los Teoremas de Euclides?

P: Uno de los objetivos es que puedan conocer los teoremas y lo segundo es enfocar la aplicación, que identifiquen los elementos que conforman dicha fórmula.

2. E: En el programa de estudio de segundo medio, actualizado el año 2011, aparece como objetivo demostrar los Teoremas de Pitágoras y de Euclides, ¿qué opina al respecto? ¿Cree usted que es necesario o primordial ese objetivo?

P: En cuanto al tema de la demostración, no es un tema que abordemos como instituto, debido a que nuestros alumnos ya vienen con serias falencias en matemáticas y como institución tenemos que lograr cerca de un 90\% de aprobación, lo cual el departamento nunca cumple, porque es una de las asignaturas en la que más reprueban. Aun así, el tema de las demostraciones por un tema de tiempo no se alcanza a abordar, más aún, este año con la situación de casi dos meses de paralización docente, es aún más complejo abordar ese tema, porque hay otros que debemos acordarnos como la semejanza de triángulos y la congruencia, y el año pasado no vimos congruencia específicamente, por ello es más complejo abordar la demostración. Debido al paro, se nos sugirió desde la unidad técnica que se abordaran los temas más relevantes que necesitaran los estudiantes para el SIMCE.

3. E: ¿Considera los Teoremas de Euclides como un contenido primordial y obligatorio 
dentro del programa de segundo medio?

P: Como primordial dentro de la enseñanza técnico profesional no en la parte obligatoria, como está dentro del programa de estudio, en los lineamientos que plantea el MINEDUC sí lo es. Pero en mi caso, no es primordial, porque teniendo semejanza de triángulos como conocimiento previo y el Teorema de Pitágoras se puede deducir alguna que otra fórmula. Y no lo hallo primordial tampoco en la enseñanza técnico profesional, porque la mayoría de mis alumnos no va a pasar la PSU, entonces no me parece tan necesario adquirir ese conocimiento y también hay razones de tiempo. Por las movilizaciones de este año no se pudo terminar la planificación formal anual que teníamos preparada.

4. E: ¿Cuántas horas en promedio cree que se le debe dedicar a este tema?

P: En promedio dependería del tipo de establecimiento y del tipo de alumnos con que contamos, en especial basándome en el caso de mis alumnos, 14 horas pedagógicas como mínimo. Eso incluye 2 horas para el conocimiento teórico y el resto para la ejercitación respectiva, ya que no se les asignan tareas para la casa con el tema de la jornada escolar completa.

5. E: En cuanto a los libros, ¿usa algún texto específico, tiene algún texto preferido?

P: Uso los Santillana y Bicentenario, además de la misma colección Santillana, el texto Geometría y Trigonometría, y también tengo de referencia un SM, que es una edición antigua y que viene con un libro de ejercicios aparte. También tengo los Santillana antiguos de los años 90, que eran de color morado, los cuales tienen la explicación matemáticamente más detallada para el docente. Pero, para este tema en particular de Pitágoras y Euclides, solo me basé en el Santillana Bicentenario.

6. E: ¿Qué opina del texto escolar que entrega el ministerio? ¿Le gusta ocuparlo?

P: Desde los últimos años me ha gustado más, ya que antiguamente teníamos la editorial Santillana, pero no era como el Bicentenario, sino que era más elevado y su uso era escaso por la poca comprensión que lograba el estudiante. Ahora, con el texto actual SM, que viene del año pasado, es mucho más estructurado porque viene con ejercicios de refuerzo y posteriormente con problemas de aplicación que van aumentando de menor a mayor el grado de dificultad, entonces es más fácil que los estudiantes puedan seguir un orden coherente de los contenidos.

7. E: ¿Cuál es el método de enseñanza que aplica a los Teoremas de Euclides? ¿Es el mismo que aplica para el resto de los contenidos?

P: En una primera parte establecer teóricamente la parte de las fórmulas, sin 
demostraciones concretas sino viendo más ejemplos de aplicaciones. Y sigo por lo general el mismo método. Lo que puede cambiar es la cantidad de tiempo teórico que dedico a la formalización de los conceptos o el uso del lenguaje, dependiendo de los temas que se traten.

8. E: En cuanto a la realización de sus clases, ¿considera que tiene un ambiente propicio como a usted le gustaría o piensa que le falta material, le falta tiempo, instrumentos didácticos?

P: El uso del tiempo propicio a veces se ve afectado, como lo pudiste observar hoy día, con las interrupciones de inspector, jefe técnico, que entregan avisos, o a veces, por los ritmos de avance de los chicos, ya que uno tiene planificado llegar hasta un cierto avance, pero no ve progreso porque no son autónomos en sus procesos. En cuanto a los materiales, lo que tenemos disponible son guías de aprendizaje, un cuadernillo de ejercicios desarrollados por el departamento y el libro del ministerio, además de las presentaciones, que son diseños dependiendo de los temas, sobre todo se usa en el área de geometría ya que como no contamos con los implementos para hacerlos a mano, es más fácil con el proyector. Lo que más cuesta es adaptarse a la optimización del tiempo, ya que son demasiados los factores que influyen, no tan solo mi planificación y orientación. Por ejemplo, hoy tuve que pedir unos datos que correspondían a jefatura, pero era una encuesta que se debía enviar a la dirección, sobre la lectura de dos boletines con información del SIMCE. Por otro lado, influyen factores externos como actos de último minuto del establecimiento, paro docente, ensayos SIMCE, licencias médicas etc.

9. E: ¿Realiza el proceso de institucionalización luego de un conocimiento nuevo?

P: De acuerdo con la teoría sí, porque por lo general, aparte de la parte teórica mezclamos lo que los alumnos van aprendiendo en la práctica, lo que se utiliza para formalizar los conceptos de una manera más matemática, que no quede solo con el uso del lenguaje más cotidiano, sino que quede la estructura de los temas.

10. E: ¿Qué tipo de problemas prefiere abordar en clases? ¿Rutinarios o abiertos?

P: Más del tipo rutinario, para que ellos puedan llegar a una solución concreta. Los problemas abiertos solo los abordamos si es que nos alcanza el tiempo en las pruebas de resolución de problemas que vienen implementándose hace un par de años acá en la institución, por el tema de la ley SEP, que son unas pruebas especiales para medir distintas habilidades, desde el manejo del lenguaje y otras habilidades superiores en la resolución de problemas. 
11. ¿Cómo controla el aprendizaje de los alumnos durante la clase?

P: Primero con el tema de la organización de la sala de clases, cuando están desordenados con el trabajo individual, el monitoreo continuo, el trabajo con apoyo del aula o del practicante, evaluaciones (aunque no en todas las clases), con el monitoreo constante de los avances o la entrega de la guía desarrollada con nota acumulativa o evaluaciones pequeñas que sean para sintetizar los contenidos vistos y tratados en una clase con nota acumulativa.

12. E: ¿Qué lenguaje prefiere usar con los estudiantes? ¿Lenguaje matemático, natural o mixto?

P: El lenguaje mixto, porque el lenguaje matemático es para la conceptualización teórica de los temas vistos, pero también se utiliza el traspaso al lenguaje cotidiano para que ellos comprendan más fácilmente lo que estamos abordando, hay que hacer ahí el uso de los sinónimos, de buscar distintas palabras para las explicaciones porque si no, no tenemos buenos resultados en el área.

\section{Análisis de las entrevistas}

En relación con los objetivos de la clase (pregunta 1), el profesor A responde que se propuso dos objetivos, uno es recordar los conceptos básicos asociados a los Teoremas de Euclides, cuestión que no se observó en la gestión de su clase. El otro, definir los conceptos y después ejemplificar y practicar. Suponemos que se refiere a explicar el enunciado del teorema mismo, lo que tampoco se observó. No obstante, él anota en la pizarra el objetivo del programa.

La profesora B señala que se plantea dos objetivos: conocer los Teoremas de Euclides y aplicar las fórmulas identificando sus elementos. Esto se pudo observar en las diapositivas y sus explicaciones.

En relación con la demostración (pregunta 2) de los Teoremas de Euclides, solicitada por el programa de segundo medio, ninguno de los dos profesores la considera, argumentando que los alumnos llegan sin las suficientes herramientas teórico-matemáticas para abordar este tipo de conocimientos, el cual requiere el desarrollo de un razonamiento lógico deductivo y de procesos de argumentación. También comentan factores externos, de carácter institucional, que afectan los tiempos escolares, ambos se refieren a circunstancias como días feriados, paros, reuniones escolares, entre otros. Estos aspectos, se les conoce como ecológicos (PINOFAN; ASSIS; CASTRO, 2015). La profesora B señala, además, que los Teoremas de Euclides 
son un tema difícil.

Para la pregunta 3, ambos profesores señalan que los Teoremas de Euclides son un contenido primordial. No obstante, la profesora B dice que para ella no lo es en la enseñanza técnico profesional.

La pregunta 4 se refiere al tiempo promedio que dedican al tema. El Profesor A señala que es relativo, él dedica entre 7 y 10 horas, y la profesora B dice mínimo 14 horas. Nos parece extraño que mencionen esa cantidad de tiempo invertido para el aprendizaje de los Teoremas de Euclides, pues con el estilo de clases que observamos, donde solamente se trataron ejercicios de aplicación de las fórmulas, pareciera que el tema se da en menor tiempo. Posiblemente, el tiempo explicitado se deba, que después de dar el tema, a la poca reflexión de los profesores sobre su práctica implementada.

Un fenómeno que señala la profesora B es que, debido a la jornada escolar completa, no se dan tareas para la casa, así que los estudiantes solo repasan los temas vistos durante su jornada de escuela y no tienen otro espacio.

En relación con el uso de textos de apoyo (pregunta 5), el profesor A no los utiliza, aunque considera buenos los libros de editorial Santillana, pero sus alumnos no tienen recursos económicos para comprarlos, mientras los textos que entrega el Ministerio de Educación no son adecuados para lo que él necesita, así que él mismo diseña sus materiales. La profesora B opina que el texto actual del Ministerio SM es mucho más estructurado, trae ejercicios de refuerzo y problemas de aplicación, lo que facilita el trabajo con los alumnos. Ella se apoya en varias colecciones de libros (Santillana, Bicentenario, una edición antigua de un SM etc.), pero no señala si sus alumnos acceden a textos del mercado, por ejemplo, los de la editorial Santillana.

En la pregunta 6, respecto a la metodología de enseñanza, el profesor A señala que su método es el expositivo, y le permite estructurar bien todo, seguido de la resolución de ejercicios. La profesora B dice, por su parte, establecer teóricamente las fórmulas, sin demostraciones, viendo ejemplos de aplicaciones, y que en general sigue el mismo método. Lo que puede cambiar es el tiempo para la formalización de los conceptos o el uso del lenguaje, dependiendo del tema.

Ambos profesores se refieren a sus modos de enseñanza en general, pero en sus clases no se observaron concretamente. No coincide lo que dicen que hacen con lo que realmente se ha observado. Ambos se refieren a la metodología tradicional de la clase axiomática (primero la teoría y luego los ejercicios de aplicación). En ambos casos, los ejercicios los resuelven ellos, produciendo con frecuencia los fenómenos del efecto Topaze y la resonancia débil o 
nula. Con ello, impiden la producción de respuestas por los alumnos y no dan lugar a la interacción entre profesor-alumno, ni entre los alumnos entre sí.

En relación con la pregunta 8, respecto al ambiente e infraestructura. El profesor A señala que no tiene mayores problemas y tiene acceso a material y guías que necesita. $\mathrm{Su}$ dificultad es la indisciplina de los alumnos que, en su opinión, no están motivados por aprender. La profesora B también señala que tiene materiales disponibles como guías de aprendizaje, un cuadernillo de ejercicios desarrollados por el departamento, el libro del ministerio, además de las facilidades para proyectar. El problema para ella son los factores externos, como actos de último minuto del establecimiento, paro docente, licencias médicas etc., que interrumpen no sólo sus clases, sino también las planificaciones y estimaciones de tiempo.

En relación con la resolución de problemas (pregunta 11) ambos profesores dicen que es difícil abordar problemas; esto debido a la falta de algunos conocimientos mínimos necesarios de los estudiantes y, por ello, prefieren abordar el tipo de ejercicios rutinarios. La profesora B señala que cuando le alcanza el tiempo puede abordar problemas abiertos, pero sólo en las pruebas en relación con la Ley de Subvención Escolar Preferencial (Ley SEP). Estas pruebas se refieren a la medición de distintas habilidades, como el cambio del lenguaje natural al lenguaje algebraico, a otras superiores en la resolución de problemas.

\section{Conclusiones}

Desde el punto de vista didáctico se constata que, en la gestión de clases de los profesores observados, ellos son los protagonistas y explican que prefieren hacer clases expositivas con formulación de preguntas, pero como lo hemos señalado, no son verdaderas preguntas, sino son superficiales y de respuesta cerrada. Esto contradice las indicaciones del Ministerio de Educación (MINEDUC) y las del Marco para Buena Enseñanza, las cuales no son consideradas por los profesores observados, y tal vez, sea una conducta general.

Los profesores no realizan un autoanálisis de sus prácticas en relación con los procesos de enseñanza y de aprendizaje. Por ejemplo, no se cuestionan sus prácticas, lo que queda en evidencia en las entrevistas, quedando conformes con sus respectivas prácticas didáctico-matemáticas. Ninguno de los dos profesores se refirió a qué aprendizaje real apuntaban. El profesor A anotó el aprendizaje esperado, pero al final de la clase no se hizo una autoevaluación en relación con el logro de objetivos - en cuanto al contenido matemático y al saber hacer -; si los medios que utilizó estuvieron apropiados; o si los alumnos tenían los 
conocimientos previos adquiridos - o en caso contrario, cuáles recordar, repasar o reforzar.

En los episodios seleccionados se observa que, en el tratamiento del objeto matemático, Teoremas de Euclides, se privilegia el punto de vista algebraico únicamente y se supone que se trataba de una clase de geometría, donde las figuras geométricas no jugaron ningún rol; es decir, ninguno de los dos profesores ha conectado las figuras geométricas para dar sentido y significado a las escrituras algebraicas.

Los enunciados de los teoremas no se presentaron geométricamente, distinguiendo las hipótesis y las tesis. Además, se utiliza el triángulo rectángulo de hipotenusa horizontal, el típico con el ángulo recto opuesto a la hipotenusa. La profesora B cambió, en uno de los ejercicios, la posición del triángulo, pero como el ejercicio estaba resuelto, a nuestro juicio, perdió valor y no se favoreció el aprendizaje. Otro resultado de aprendizaje se habría obtenido si los alumnos hubieran podido distinguir la posición de las proyecciones sobre la hipotenusa, el rol de las notaciones hubiera surgido y el aprendizaje de los Teoremas de Euclides se habría favorecido.

Por otro lado, los profesores piensan que ellos siguen el Programa y están conscientes que pueden adaptarlo, pero no es lo que se aprecia en sus gestiones de clases; también el hecho de realizar preguntas a los alumnos, para ellos es realizar una clase dialogada, pero ninguno de los dos interactúa verdaderamente con los alumnos. Cuando un alumno ha preguntado a la profesora B si hay que copiar también la figura, ella le responde obvio. Pero el significado de esa pregunta tiene que ver con el contrato didáctico (algunos autores lo llaman normas, e.g., MOLINA, 2019) y particularmente, con la responsabilidad del profesor, al explicitar a los alumnos su tarea a realizar. La observación de la clase deja en evidencia que los alumnos no tuvieron otra responsabilidad que estar atentos al trabajo del profesor en la pizarra, y seguirlo contestando a preguntas superficiales.

Por su parte, el profesor A no toma en cuenta la intervención del alumno A5, cuando responde correctamente en el instante (9) del Episodio 3. ¿Por qué? Tal vez porque no quiso perder el hilo de su razonamiento al desarrollar el ejercicio, o porque simplemente no lo escuchó. En todo caso, a nuestro juicio, estos hechos están relacionados con la falta de interacción con los alumnos y la falta de conciencia de que las actividades de los alumnos son las protagónicas (PINO-FAN; ASSIS; GODINO, 2015).

En síntesis, ninguno de los dos profesores ha favorecido la interacción con sus alumnos, quedando en evidencia el fenómeno de resonancia nula o ausencia de resonancia; además, tampoco previeron actividades matemáticas para los alumnos que les permitiera elaborar respuestas de manera autónoma. Lo anterior no sólo deja en evidencia falencias en la 
preparación de las clases, sino también en las concepciones de las matemáticas escolares y de las especificidades del aprendizaje matemático.

Este trabajo, a nuestro juicio, deja pistas abiertas para diseñar posteriores reciclajes de profesores, es decir, espacios de profesionalización donde los docentes apoyados en marcos teóricos y un formador miran su experiencia práctica para reflexionar sobre sus conocimientos, actitudes y habilidades que poseen.

Por otra parte, este artículo presenta una doble finalidad, por un lado, que el profesorado reconozca y tome conciencia de la existencia de las paradojas didácticas y sus consecuencias negativas con relación al aprendizaje y, por otro lado, entregar evidencia empírica de la gestión de clases, cuestión que queda invisible en la mayoría de las investigaciones sobre las prácticas pedagógicas.

Finalmente, con el trabajo realizado se evidencia la necesidad de generar espacios para que los profesores reflexionen sobre sus prácticas pedagógicas o didáctico-matemáticas (PINO-FAN; ASSIS; CASTRO, 2015; CASTRO; PINO-FAN; VELÁSQUEZECHAVARRÍA, 2018). Para ello, es necesario disponer de herramientas teóricometodológicas que permitan tanto sistematizar tal reflexión, de manera que se contemplen las distintas dimensiones involucradas en los procesos de enseñanza y aprendizaje de las matemáticas (matemática, cognitiva, efectiva, ecológica, medios, interacciones etc.), como realizar análisis más finos que contribuyan en la toma de decisiones dentro del aula (CASTRO; PINO-FAN; VELÁSQUEZ-ECHAVARRÍA, 2018).

Una línea de investigación que queda abierta es establecer estudios de networking entre la TSD y modelos del conocimiento del profesor de matemáticas, tal como el Modelo del Conocimiento Didáctico-Matemático - CDM - (PINO-FAN; GODINO, 2015), o la TSD con el sistema de normas propuesto por el EOS, tal como el realizado por Molina (2019) en su estudio, los cuales podrían permitir análisis más profundos de la gestión dinámica de clases.

\section{Agradecimientos}

Este trabajo ha sido desarrollado en el marco del Proyecto de Investigación Fondecyt $N^{\circ} 1200005$ financiado por la Agencia Nacional de Investigación y Desarrollo (ANID) de Chile.

\section{Referencias}

BREDA, A.; FONT, V.; PINO-FAN, L. Criterios valorativos y normativos en la Didáctica de las 
Matemáticas: el caso del constructo idoneidad didáctica. Bolema, Rio Claro, v. 32, n. 60, p. 255-278. 2018.

BROUSSEAU, G. Fondéments et méthodes de la didactiques des mathématiques. Recherches en Didactique des Mathématiques, Grenoble, v. 7, n. 2, p. 33-115, 1986.

BROUSSEAU, G. Les différents rôles du maître. Bulletin de l'A.M.Q, Québec, n. 23, p. 14-24, 1988a.

BROUSSEAU, G. Le contrat didactique: le milieu. Recherches en Didactique des Mathématiques, Grenoble, n. 9, p. 309-333, 1988b.

BROUSSEAU, G. ¿Qué pueden aportar a los enseñantes los diferentes enfoques de la didáctica de las matemáticas? Enseñanza de las Ciencias, Barcelona, v. 8, n. 3, p. 259-267, 1990.

BROUSSEAU, G. L'enseignant dans la théorie des situations didactiques: 1. Structure et fonctionnement du système didactique. Actes de la VIII Ecole d'été de didactique des mathématiques St-Sauves d'Auvergne, Francia, p. 3-46. 1995.

BROUSSEAU, G. La Théorie des situations didactiques. Grenoble: La Pensée Sauvage, 1998.

BROUSSEAU, G. Educación y Didáctica de las Matemáticas. Educación Matemática, Ciudad de México, v. 12, n. 1, p. 5-38, 2000.

BROUSSEAU, G. Theory of Didactical Situations in Mathematics: Didactique des Mathématiques, 1970-1990. Dordrecht, Springer. 2002.

BROUSSEAU, G. Notes on the observation of classroom practices, International Congress on Mathematical Education 11, Monterrey, n. 4, p. 1-7. 2008.

CASTRO, W. F.; PINO-FAN, L.; VELÁSQUEZ-ECHAVARRÍA, H. A proposal to enhance preservice teachers noticing. Eurasia Journal of Mathematics, Science \& Technology Education, London, v. 14, n. 11, p. 1569-1585, 2018.

COMITI, C.; GRENIER, D.; MARGOLINAS, C.; Niveaux de connaissances en jeu lors d'interactions en situation de classe et modélisation de phénomènes didactiques. In: ARSAC, G.; GRÉA, J; GRENIER, D.; TIBERGHIEN, A. (ed.). Différents types de savoirs et leur articulation, Grenoble: La Pensée Sauvage, 1995. p. 92-113.

FORTUNY, J.; RODRÍGUEZ, R. Aprender a mirar con sentido: facilitar la interpretación de las interacciones en el aula. Avances de investigación en Educación Matemática, Badajoz, v. 1 n. 1, p.23-27, 2012.

HERSANT, M. Facette épistémologique et facette sociale du contrat didactique: une distinction pour mieux caractériser la relation contrat milieu, l'action de l'enseignant et l'activité potentielle des élèves. Recherches en didactique des mathématiques, Grenoble, v. 34, n. 1, p. 9-31, 2014.

LAPARRA, M.; MARGOLINAS, C. Quand les maîtres contribuent à leur insu à renforcer les difficultés des élèves. In: ROCHEX, J.-Y.; CRINON, R. (ed.). La construction des inégalités scolaires. Rennes: Presses Universitaires de Rennes, 2011. p. 111-130.

LLINARES, S.; VALLS, J.; The building of pre-service primary teachers' knowledge of mathematics teaching: interaction and online video case studies. Instructional Science, Dordrecht, v. 37, n. 3, p. 247-271, 2009. 
MARGOLINAS, C. Eléments pour l'analyse du rôle du maître: les phases de conclusion. Recherches en Didactique des Mathematiques, Grenoble, v. 12, n.1, p. 113-158, 1992.

MARGOLINAS, C. De l'importance du vrai et du faux dans la classe de mathématiques. Grenoble: La Pensée Sauvage, 1993. Disponible en: https://hal.archives-ouvertes.fr/hal-00779138. Acceso en: 18 oct. 2018.

MARGOLINAS, C. La structuration du milieu et ses apports dans l'analyse a posteriori des situation, Recherches en didactique des mathématiques, Grenoble, n. 1, p. 89-102. 1995. Disponible en: https://halshs.archives-ouvertes.fr/halshs-00418815/document. Acceso en: 9 dic. 2017.

MINISTERIO DE EDUCACIÓN - MINEDUC. Matemáticas educación media. Programa de Estudio Matemática II Medio. Santiago: Unidad de Currículum y Evaluación, 2011.

MOLINA, O. Sistema de normas que influyen en procesos de argumentación: un curso de geometría del espacio como escenario de investigación. 2019. Tesis (Doctorado en Educación Matemática) - Departamento de Ciencias Exactas, Universidad de Los Lagos, Osorno, 2019.

PERRIN-GLORIAN, M. Théorie des situations didactiques: naissance, développement, perspectives . En: Atigue, M. ; Gras, R, ; Laborde, C. ; Tavignot, P. (ed.). Vingt ans de didactique des mathématiques en France. París: La Pensée Sauvage, 1994. p. 97-147.

PINO-FAN, L.; ASSIS, A.; CASTRO, W. F. Towards a methodology for the characterization of teachers' didactic-mathematical knowledge. Eurasia Journal of Mathematics, Science \& Technology Education, London, v. 11, n. 6, p. 1429-1456, 2015.

PINO-FAN, L.; ASSIS, A.; GODINO, J. Análisis del proceso de acoplamiento entre las facetas epistémica y cognitiva del conocimiento matemático en el contexto de una tarea exploratorioinvestigativa sobre patrones. Educación Matemática, Ciudad de México, v. 27, n.1, p. 37-64. 2015.

PINO-FAN, L.; GODINO, J. D. Perspectiva ampliada del conocimiento didáctico-matemático del profesor. Paradigma, Maracay, v. 36, n.1, p. 87-109, 2015.

TCHOSHANOV, M.; Relationship between teacher knowledge of concepts and connections, teaching practice, and student achievement in middle grades mathematics. Educational studies in mathematics, Utrecht, v. 76, n. 2, p. 141-164, 2011.

TROUCHE, L.; GITIRANA, V.; MIYAKAWA, T.; PEPIN, B.; WANG, C. Studying mathematics teachers interactions with curriculum materials through different lenses: Towards a deeper understanding of the processes at stake. International Journal of Educational Research, Belfast, v. 93, p. 53-6, 2019. 